\title{
全方位多层次立体化的应用化学专业实验教学模式的构建与实践
}

黄健涵, 陈立妙, 曾冬铭, 李亚娟, 桑商斌, 潘春跃, 王海燕, 唐俊涛, 宋相志, 王一凡, 罗一鸣, 刘又年 ${ }^{*}$

中南大学化学化工学院, 长沙 410083

摘要: 在新工科和国家一流本科专业建设大背景下, 针对当前应用化学专业学生实验教学中能力和综合素质培养存在的 问题, 结合中南大学应用化学专业的自身特色与优势, 创新教育理念, 按照目标导向的方式对实验教学体系、教学内容 和教学方法等方面进行系统性的教学改革与实践, 建立了全方位多层次立体化的应用化学专业实验教学新模式, 取得了 良好的教学效果。

关键词: 应用化学; 实验教学; 教学模式; 多层次; 立体化

中图分类号: G64; 06

\section{Construction and Practice of an All-Round, Multilevel, and Three- Dimensional Laboratory Teaching Model in Applied Chemistry}

Jianhan Huang, Limiao Chen, Dongming Zeng, Yajuan Li, Shangbin Sang, Chunyue Pan, Haiyan Wang, Juntao Tang, Xiangzhi Song, Yifan Wang, Yiming Luo, Younian Liu * College of Chemistry and Chemical Engineering, Central South University, Changsha 410083, China.

\begin{abstract}
Based on the background of "emerging engineering education" and "first-class majors", we focused on solving the problems of the ability and quality cultivation of applied chemistry undergraduates in laboratory teaching. According to the characteristics and advantages of applied chemistry major in Central South University, we had a goaloriented educational reform in laboratory teaching system, teaching contents and teaching methods. As a result, we had constructed a new all-round, multilevel, and three-dimensional laboratory teaching system in applied chemistry, and this innovative practice teaching system has achieved good teaching effects.
\end{abstract}

Key Words: Applied chemistry; Laboratory teaching; Teaching model; Multilevel; Three-dimensional

实验教学是应用化学专业教学的重要组成部分, 在专业人才培养中发挥着基础性、关键性作 用 ${ }^{[1]}$ 。当前, 新工科和国家一流本科专业建设的大环境对应用化学专业实验教学提出了新要求, 应 用化学专业实验教学如何结合专业特点, 创新教育理念, 贯彻 “强化基础、注重综合、突出创新、 培养能力、提高素质” 的改革方针, 构建新的实验教学模式, 更新实验教学体系, 实现以学生为主 体, 激发学生学习的主动性和创造性, 培养学生的创新能力和综合素质, 实现实验教学的线上线下 融合、虚实互补的多元协同混合式教学，体现信息技术与实验教学的深度融合，助推应用化学专业 人才培养的内涵式发展, 是新时代提出的新命题 ${ }^{[2,3]}$ 。对标新工科和一流本科专业建设的要求, 中南

收稿: 2021-05-10; 录用: 2021-06-17; 网络发表: 2021-07-01

“通讯作者, Email: liuyounian@csu.edu.cn

基金资助: 国家一流本科专业建设点(教高厅函[2021] 7号); 湖南省一流本科专业建设点(湘教通[2019] 138号); 中南大学教育教学改革项目 (2021jy068) 
大学应用化学专业立足自身定位, 在国家级工科大学化学基础课教学基地、国家级化学实验教学示 范中心、国家级矿冶工程化学虚拟仿真实验教学中心、国家级一流课程等本科教育质量工程项目建 设的引领下, 秉承 “宽口径、厚基础、凸特色、重创新” 的办学方针 ${ }^{[4]}$, 坚持 “学生中心、产出导向、 持续改进” 的理念, 打破以往的惯性思维, 按照目标导向的方式对实验教学体系、教学内容和教学 方法等进行系统性的教学改革, 建立了全方位多层次立体化的应用化学专业实验教学新模式, 大幅 度提升了实验教学效果, 丰富了应用化学专业建设内涵。

\section{1 创新教育理念, 牢固树立人才培养的中心地位}

当前, 相对于理论教学, 化学实验教学的重视程度还有待进一步加强, 重理论轻实验的现象仍 然存在, 实验课程中学生 “做的不少, 会的不多” 现象还比较严重, 实验教学目标的达成还不够理 想 ${ }^{[1]}$ 。为此, 我们创新教育理念, 将实验教学由理论教学的 “从属” 转变为人才培养的 “重要组成部 分”, 2016年的培养方案将实践性教学环节修改到占总学分的 $34.3 \%$ (总学分 179.5 ), 实验教学占实践 性教学环节的 $80.0 \%$, 凸显了实验教学的中心地位。实验教学从后台走向前台, 由 “从属” 转变为 “主 导”, 成为创新教学的主体角色, 让实验教学成为培养学生兴趣、心向和情感的良好场所。同时, 以 人为本, 突出学生为主体、教师为主导, 抓好实验课前预习、课中讨论、课后总结报告的三环节。通 过学生主持课中实验讨论等教学方法, 激发学生学习的主动性和创造性, 突出研究与探索, 培养学 生的创新精神、创新思维和创新能力, 实现技能训练和智能培养同步发展。严格要求, 重视原始实 验记录, 注重科学精神教育、人文素养教育和终身学习能力培养。另外, 充分利用互联网、虚拟现 实、云计算、大数据等信息化技术, 实现实验教学的线上线下融合、虚实互补, 促成多元协同混合 式教学, 推动信息技术与实验教学的深度融合, 构建以 “思想政治引领、核心价值塑造和创新能力 培养”为主线的以本为本的实验教学模式 ${ }^{[4]}$ 。

\section{2 构建全方位多层次立体化的实验教学体系}

以专业特色方向为导向, 以 “基本要求+特色” 为出发点, 重组 “四层次一体化” 实验教学体系, 理工结合, 线上线下融合, 虚实互补和创新创业贯穿, 构建了以基础实验、综合实验、科学训练、专 题实验和创新创业为纽带的全方位多层次立体化的实验教学体系, 并基于产出导向理念, 不断优化, 持续改进教学方式, 突出实验教学体系对专业人才培养的重要支撑作用。

\section{1 全方位}

(1) 以思想政治教育为引领。将思想政治教育贯穿实验教学全过程, 挖掘实验课程中的思政元 素, 发挥实验教学的育人功能, 将实验课程与育人有机结合, 达到 “润物细无声” 的育人效果。例 如, 在基础化学实验中, 强调实验安全和环境保护; 通过学习 “三废” 处理, 树立 “生态兴则文明 兴” 的理念, 了解蓝天白云保卫战等国家政策, 践行清洁生产、科学治污和可持续发展 ${ }^{[5]}$ 。在无机化 学实验 “氯化钠的提纯” “胆矾的精制” 等实验中, 培养学生诚信、友善及互助的价值观, 并让学生 树立环保观念。在分析化学实验中, 所有的分析检测都需要进行至少三次的平行试验, 在数据处理 中对数字的取舍以及运算都有严格的要求, 培养学生严谨的科研态度和严密的逻辑思维 ${ }^{[6]}$ 。

（2）以能力培养为导向。理顺实验教学体系, 在夯实四大基础实验的基础上, 强化专业实验, 设置专业综合实验、专业科学训练、专业专题实验和毕业论文环节。教学内容体现综合性、设计性 和创新性, 教学方法关注规范, 注重结果, 强调系统设计、分析综合和判断创新。注重化学与化工 相结合, 理论与应用相结合, 书本与实验相结合, 研究与创新相结合, 真实与虚拟相结合, 探究与 工艺相结合。建立制备-分离-成型-应用、制备-提纯-表征-性能、制备-应用-环保等多工艺路线, 将科研的手段、技能和方法融入实验项目中, 将工艺条件的摸索融入实验过程中, 助推 “科研成果 进实验课堂、科研进实验室、科研进实验教材”, 着力培养学生的创新意识, 引导学生自主学习、自 我发展。 
(3) 以综合素质培养为目标。(1) 依托国家级实验教学示范中心、矿冶工程化学国家级虚拟仿 真实验教学中心等平台, 通过开设名师论坛、企业交流、校友讲座等方式, 培养学生的独立人格、 思辨精神、专业视野、职业规范等综合素质。(2) 利用国家工科基础课程化学教学基地等平台, 以教 师的科研项目和大学生创新训练项目为依托, 科教融合, 协同育人, 科学设计学生的科学研究途径, 由浅入深、由简单到复杂、由合作到独立, 一步一步地完成科学研究过程, 培养学生的科研能力和 创新意识, 实现科研素养、能力和综合素质的飞跃。(3) 利用 “校-院-企” 的相互协作, 联合建立学 生校外创新创业教育基地和实践基地, 为学生的工程训练、专业实习、毕业论文等提供坚实的平台, 专业聘请了 30 余位企业专家担任兼职教师, 参与专业选修课、科学训练、专题实验、实习及毕业论 文的教学, 丰富了专业实验教学的内容, 着力培养学生的工程能力与创业意识。

\section{2 多层次}

（1）重组基础实验, 夯实基础。以优化课程结构为先导, 将四门专业基础实验课程的有关原理 和方法整合, 形成以物质制备为主线, 基本操作、物理化学实验研究方法和现代仪器分析手段为基 本内容的基础化学实验课程体系。以方法论和学科基础知识的综合运用为原则, 重组实验项目和实 验内容, 培养学生扎实的化学实验基本技能。如无机化学实验中将化学基本操作、实验研究方法和 现代分析手段等有机融合, 主要涉及基本操作实验, 包括化学实验基本知识、无机物分离和纯化方 法、基本理化数据测定方法、常规无机物制备原理和方法、常见定量分析方法以及与元素化学原理 相关的沉淀反应、颜色反应等定性分析方法, 给学生呈现一个比较完整的化学实验基本知识体系。 有机化学实验中, 强化蒸馏、重结晶、萃取与分液、薄板层析、柱层析、干燥、测折射率、测熔点、 分馏、水蒸气蒸馏、分水器的使用、旋转蒸发仪的使用、无水无氧技术等常规操作, 熟悉基本的有 机化合物如: 乙酰苯胺、乙酰水杨酸、安息香、苯甲醇和苯甲酸、乙酰二茂铁、对氨基苯甲酸以及 对氨基苯甲酸乙酯的制备方法。通过训练学生的基本实验操作, 培养学生的实验操作能力、分析问 题和解决问题的能力, 同时加深学生对基本化学原理的理解, 为今后进一步深造和开展实际工作或 进行化学相关科学研究奠定良好的实验基础。

(2) 整合综合实验, 打通科研生产全流程。我们优化了专业综合实验体系, 编写了一本全新的 应用化学专业综合实验教材《化学化工综合实验》, 在第六学期开设64学时的应用化学综合实验。 实验内容将无机化学、有机化学、分析化学、物理化学等基础化学知识及无机合成、有机合成、生 物化学、环境化学、精细化学品化学、电化学、材料化学、分离工程与技术等多门专业基础知识有 机结合, 并结合我校应用化学专业在有色金属资源化学方面的特色与优势, 精心挑选了 “ $\mathrm{BiOX}(\mathrm{X}=$ $\mathrm{ClBrI}$ ) 的制备表征及其光催化性能研究” “二氢吡啶类心血管药物硝苯地平的合成与结构表 征” “超级电容器用导电聚合物的电化学合成及性能测试” “水系锌离子扣式电池组装及电化学性 能测试” “GC-MS和LC-MS联合表征血浆中小分子化合物” “食品危害因子的拉曼快速检测及基 底材料的制备” “聚磷酸铵的制备及阻燃性能测试” “电解法印染废水脱色及COD的测定” “用 液膜分离法处理含铜溶液” “氯化氢催化氧化及反应动力学虚拟仿真实验” 和 “高硫高砷金精矿的 氧化焙烧-氧化浸出虚拟仿真实验” 等 11 个实验项目供学生选择。新的实验内容: (1) 紧扣当今现实 以及企业的技术难题, 注重理论、实验与社会需求之间的衔接, 使学生真正体会到化学创造世界, 激励学生从事化学的热情, 拓宽学生的知识面。(2) 强调项目的综合性, 做到合成-表征-应用的有机 结合, 克服 “照方抓药” 的弊端以及传统实验中方法与技能互不相干的脱节现象。(3) 体现项目的研 究性, 实验内容涉及材料的配比、结构与性能的构效关系, 同时引入新的检测手段及仪器设备, 对 大型仪器的使用进行培训。(4) 拓展项目的应用, 实现由基础向应用的转化, 涉及材料的应用性能, 如催化、发光、电化学、阻燃等特性。(5) 体现项目的工程放大, 提供离子交换、膜分离和超临界萃 取技术, 引入成型中的喷雾干燥、反应中的固定床催化等实验项目, 体现实验室的小试与工业生产 的衔接, 对工艺的设备进行参数调试, 获取工艺数据, 为进一步工艺设计提供基本参数。(6) 践行绿色 化学理念。通过开展微量实验、妥善处理实验室 “三废”、采用仿真技术辅助实验教学等手段, 降低 
教育成本、减轻环境污染, 提高学生的环保意识, 培养学生的社会责任感。例如, 基于当前印染废 水的治理难题, 引入了 “电解法印染废水脱色及COD的测定” 实验, 让学生掌握粒子群电极电解法 脱色法过程中活性炭的吸附富集、电解作用, 催化分解作用等作用的协同, 进一步组装脱色反应器, 对人造的亚甲基蓝溶液通电条件下脱色, 测定实验前后亚甲基蓝的 COD值, 掌握电解法印染废水脱 色技术和COD测定技术。

(3) 导入科学训练, 培养科学研究方法。依托本专业的有色金属材料科学与工程教育部重点实 验室、化学电源湖南省重点实验室、微纳材料界面科学湖南省重点实验室和共建的有稀有金属分离 与综合利用国家重点实验室、先进储能材料国家工程研究中心、国家贵金属材料工程技术研究中心 和湖南华菱线缆股份有限公司国家技术中心等七大科研平台, 为学生在第七学期开设 32 学时的科学 训练课程。一方面, 我们将教师的科研项目、科研成果转化为实验教学内容, 如 “聚合硫酸铁的制 备及废水的处理” 是曾冬铭教授的科研成果转化项目, “ $\mathrm{ZnO}$ 粉体及苂光、压敏、气敏性能的测定” 是陈立妙教授的科研项目; 另一方面进行开放式教学, 开发了 “聚乙烯醇缩甲醛胶的制备、游离甲 醛的消除与测定” “硬脂酸锌、钡、镉PVC热稳定剂的制备及性能” “聚醋酸乙烯酯的制备及粘结 性能测试” 和 “洗涤剂、雪花膏的研制及性能” 等实验项目, 让学生在专业实验室进行科学训练。这 些科学训练在教师的指导下由学生自主完成。(1) 学生依据实验项目, 提出问题, 搜集资料, 探究解 决的途径。(2) 归纳研究方案, 提出假设, 进行可行性论证。(3) 制定研究方案, 进行实验, 按计划 进行科学探究。(4) 规范操作, 收集数据。(5) 数据处理, 推理论证, 获得实验结论, 验证猜想与假 设。在科学训练完成后, 还要求学生就自己的科学训练内容参照《化学学报》杂志的格式撰写一篇 科学小论文, 让学生熟悉科研论文的一般书写格式和要求。

(4) 切入专题实验, 理顺科学研究思路。我们在第七学期的最后三周集中切入专题实验, 要求学 生进入科研团队教师的实验室选题, 结合所学的基础知识、专业知识和 “文献检索” “专业英语” 等专业课程, 就某一个专题进行文献检索, 查阅相关研究领域的最新进展, 写出文献综述报告。然 后在教师指导下, 制订实验方案, 自行进行实验的组织, 包括化学药品的准备、仪器的组装、实验 的操作、数据处理、小论文的写作等。通过专题实验, 学生的查阅文献能力、文献综述能力、实验 的组织与操作能力以及分析问题和解决问题的能力都得到了有效训练, 为第八学期毕业论文工作的 顺利开展打下了良好基础。在此过程中, (1) 专业实验室、科研实验室和科研平台向学生 “三开放” (时间、空间和内容上开放), 鼓励学生早期参与导师课题, 通过研讨式与探究式学习, 培养学生良好 的科学作风和习惯, 培养学生的原始创新能力; (2) 鼓励学生参加高水平国内外学科竞赛, 如中国 “互 联网+” 大学生创新创业大赛、全国大学生化学实验创新设计竞赛、全国大学生化学实验邀请赛等; (3) 鼓励学生申请各类创新创业项目, 通过项目驱动, 寻找自己的兴趣点与关注的研究热点; (4) 鼓 励学生参加开放性实验项目, 利用学校和学院的公共平台培养学生的创新能力; (5) 鼓励学生参与学 院与比亚迪、格林美、鹏辉、长远锂科等 20 余家上市企业联合培养创新人才所提出的创新项目, 促 进产学研的发展。

\section{3 立体化}

(1) 理工结合。中南大学应用化学专业是介于化学和化工之间的应用理科专业。以新工科建设 为契机, 主动适应新技术、新业态、新产业的发展, 强化理科基础, 强调理科向工科的延伸, 瞄准 产业发展需要, 培养综合素质高、创新能力强的应用型人才。因此, 在实验环节中注重: (1) 既要完 成一般的化学实验, 也要完成 “化工原理实验”, 突出工程实验的特点, 强调基础理论与工程实践 相结合, 强调工程观念的培养, 注重化工实验的共性问题; (2) 既有专业综合实验, 又有工艺实验; (3) 既要进行化学理论、结构与性能方面的探讨, 又要进行化工计算与设计; (4) 实验完成后既要写 实验报告、科学论文, 又要做工艺设计与工程设计。强化化工应用意识, 加强向工科的渗透, 实现 理工结合。

(2) 虚实互补。将实验教学与微课、慕课、虚拟仿真等信息技术深度融合, 通过线上线下融合、 
虚实互补的协同混合式教学。利用国家级矿冶工程化学虚拟仿真实验教学中心平台, 开发了 “氯化 氢催化氧化及反应动力学虚拟仿真实验” “锂硫电池材料制备、软包电池制作与性能测试” “高硫 高砷金精矿的氧化焙烧-氧化浸出虚拟仿真实验” “火法炼铜虚拟仿真实验” 等 28 个虚拟仿真项目 供学生选择实验。自2016年以来, 在线实验达 10300 余人次。

(3) 创新创业贯穿。中南大学在全国高校中首次提出 “建设创新型大学” 的理念, 应用化学专 业将创新创业教育落实在实验教学全过程, 要求学生参加课外活动、科研实践和社会实践, 必须取 得至少6学分。(1) 激发学生创新思维。瞄准科学前沿, 通过精选实验教材和新编特色实验教材, 将 学科前沿知识和最新科研成果融入到实验教学, 拓展实验教学内容。例如, 刘洪涛教授主讲 “应用 化学综合实验”, 结合中南大学在新能源材料领域的特色与优势, 将最新电极制备与性能评价方法 的成果作为案例, 融入实验教学。钟宏教授主讲 “化工计算与设计”, 引入团队的国家科技进步二 等奖项目 “硫化矿新型高效捕收剂的合成技术与浮选应用”, 把教学内容推向学科前沿。建立学生设 定课题、查阅资料、设定方案、解决问题的教学方法和组织形式, 激发学生的科学创新思维, 科学 研究反哺实验教学。(2) 培养学生创新能力。设计探索性实验, 熟悉科学研究的方法和过程, 引导学 生尽早从事科研活动, 加入导师科研团队, 参与导师科研项目 ${ }^{[7]}$ 。例如, 王平山教授是中组部国家级 人才, 主讲 “应用化学综合实验”, 在实验教学过程中, 把自己的丰富科研经验结合专业知识传授 给学生, 让学生自然而然明白了一流的科研应该怎样做。(3) 鼓励学生积极申报学校创新研究项目, 以课题研究为载体, 培养学生的创新意识和动手能力。(4) 以赛促学, 以学带赛, 赛学结合, 提升 学生实践能力。每年4月, 学院开设专题讲座和技术培训, 指导学生参加各类实验训练和竞赛活动, 锻炼学生综合运用专业知识以及安全、环保、经济评价等知识的综合能力。学生自行组建团队, 进 行项目设计, 参加国内外学科竞赛。通过学科竞赛提高学生的创新能力, 实现 “教学改革-学科竞赛-科 技创新”的互促。

\section{3 取得的主要成果}

构建的全方位多层次立体化的应用化学专业实验教学新模式助推了中南大学应用化学专业的内 涵式发展, 近3年来获 “国家大学生创新创业训练计划 “1311' 体系创新及十年实践” “ “四三式” 创新创业人才培养模式的研究与实践” 等国家教学成果奖 2 项, “近化学类专业化学化工课程信息 化混合式教学的研究与实践” 等省级教学成果奖多项; 建立 2 个智慧教室; 获 “氯化氢催化氧化虚拟 仿真实验” “高硫高砷金精矿的氧化焙烧——氰化浸出虚拟仿真实验” 等国家级虚拟仿真实验项目 2项, “火法炼铜虚拟仿真实验” 等湖南省虚拟仿真实验项目 3 项; 建设基础化学、大学化学等国家 级 “金课” 2门; 获工科大学化学、工科大学化学实验等国家精品资源共享课4门, “有机化学-结构、 反应、机理、合成” 等湖南省一流课程 $3 门$ 门 出版《化学化工综合实验》《无机化学实验》《现代分析 化学试验》(第2版)等实验教材6部。教师教学能力不断提升, 获第一届全国高等学校物理化学(含实 验)教学微课大赛、湖南省首届微课大赛等教学奖励 15 项。学生培养质量明显提高, 近 5 年学科竞赛 参与率 $50 \%$ 以上, 获中国 “互联网+” 大学生创新创业大赛、全国大学生化学实验创新设计竞赛、全 国大学生化学实验邀请赛、全国大学生化工实验大赛等国家级竞赛奖励 58 项, 其中国家A类竞赛奖 励 30 余项。创新创业项目参与率 $60 \%$ 以上, 承担国家级创新创业项目 70 余项, 发表学术论文 200 余篇; 每年 $15 \%$ 左右的学生毕业论文获校级优秀毕业论文; 毕业生升学深造率年均 $43.0 \%$ 。毕业生适应社 会需求, 学生就业率年均 $98.0 \%$ 。近 5 年调查结果显示, 毕业生对本专业人才培养质量评价高 $(94.4 \%)$, 用人单位对毕业生满意。专业结构持续优化, 先后获得了国家特色专业(2009)、国家综合改革试点专 业(2015)和国家一流本科专业建设点(2020), 专业排名稳居全国同类专业前 $3 \%$ 。

\section{4 结语}

在新工科和国家一流本科专业建设大背景下, 针对当前应用化学专业学生实验教学中实验课程 
建设内涵不够、重理论轻实验、学生技能训练与综合素质培养不协调等问题, 创新教育理念, 将思 想政治教育贯穿实验教学全过程, 将创新创业教育贯穿实验教学全过程, 突出实验教学在创新人才 培养中的主导作用, 推进教学设计科学化、教学内容精细化、教学手段灵活化、教学方法多样化, 构建了全方位多层次立体化的应用化学专业实验教学新模式, 将训练实验技能、训练知识转移能力、 训练创新创业意识等环节相互融合, 从课内延伸到课外, 提高了学生的动手能力和综合素质, 提升 了学生自主学习、探究问题和解决问题的能力, 助推应用化学专业人才培养的内涵式发展。

\section{参 考 文 献}

[1] 张树永, 朱亚先, 张剑荣. 大学化学, 2018, 33 (10), 1 .

[2] 张树永, 朱亚先, 霍冀川, 宋丽娟, 徐华龙, 郑兰荪. 大学化学, 2020, 35 (10), 6 .

[3] 张树永, 朱亚先. 大学化学, 2021, 36 (2), 2012036.

[4] 黄健涵, 陈立妙, 李亚娟, 王海燕, 唐俊涛, 桑商斌, 潘春跃, 宋相志, 罗一鸣, 刘又年. 大学化学, 2021, 36 (11), 2104015.

[5] 夏晓红. 大学化学, 2021, 36 (5), 2009030.

[6] 张玉荣, 袁耀锋. 大学化学, 2021, 36 (3), 2007057.

[7] 郭栋才, 王玉枝, 李永军, 吴朝阳, 刘浩然, 蔡双莲, 高娜, 蔡炽. 大学化学, 2019, 34 (4), 1. 